\title{
“EXPERIENCIAS UNIVERSITARIAS DE ENSEÑANZAS A DISTANCIA. PRAXIS, VISIONES Y HORIZONTES"
}

\author{
Virginia Scotta (Universidad Nacional de Rosario)* \\ virscotta@gmail.com
}

Recibido: 30/08/13 Aceptado: 15/11/13

Comentarios sobre el libro de Copertari, S y Morelli S, (comp), (2013). EXPERIENCIAS UNIVERSITARIAS DE ENSEÑANZAS A DISTANCIA. Praxis, visiones y horizontes. 2013, Rosario, Laborde Editor.

Experiencias Universitarias... es un libro compilado por Susana Copertari y Silvia Morelli, y prologado por Sonia Concari. Ha sido editado por Laborde, en la ciudad de Rosario en marzo del 2013. A lo largo de ocho capítulos, un equipo de investigadores de la Universidad Nacional de Rosario, analiza desde la década del 90 hasta la actualidad, experiencias desarrolladas en distintas Facultades, relacionadas a la práctica de la enseñanza universitaria con modalidad a distancia.

El gran avance de la informática, y con esta, de la educación a distancia, trajeron aparejadas nuevas formas de conocer, de relacionarnos, de investigar e incluso de pensar. La Universidad Nacional de Rosario (UNR) no ha sido ajena al impacto de esta revolución tecnológica. Un sinnúmero de experiencias se fueron desarrollando a través de cátedras, cursos y carreras, donde confluyeron alternativas pedagógicas y didácticas propias de la modalidad, pero también procesos de transformación generados por el avance de las tecnologías digitales.

En este sentido, los autores nos guían a través de los distintos capítulos, por un recorrido histórico y analítico permitiéndonos recuperar la labor de do-

\footnotetext{
* Magister en Desarrollo Económico Local, Universidad Autónoma de Madrid, UAM. Prof. Universitaria Universidad Abierta Interamericana, UAI

Lic en Ciencia Política, Facultad de Ciencia Política y Relaciones Internacionales, UNR Prof Adjunta Energía y Sostenibilidad, Maestría en Energía para el Desarrollo Sostenible, Escuela de Posgrado y Educación Continua, Facultad de Ciencias Exactas, Ingeniería y Agrimensura, UNR.
} 
centes e investigadores que, desde la interdisciplinariedad, fueron construyendo y reconstruyendo la educación a distancia y su significación en la Universidad.

Así, Elsie Laurino en Historia de la Educación a Distancia. Continuidades y discontinuidades en la Universidad Nacional de Rosario, nos introduce en diferentes espacios institucionales destinados a configurar una relación no presencial entre docentes y estudiantes.

En La distancia en la educación universitaria, Silvia Morelli, nos propone una reflexión pedagógica tendiente a abrazar la modalidad como facilitadora de la enseñanza aprendizaje, pero concebida desde una perspectiva de educación integradora, democrática y alternativa.

Susana Copertari y Ana María Trotini en Experiencias a distancia universitarias sin distancia. Hacia una pedagogía de la virtualización, nos ayudan a reflexionar sobre el rol de las TIC en las nuevas formas de aprender y enseñar, desde una mirada que nos lleva a comprender la educación y la comunicación como conceptos que se "entrelazan en una praxis sociocultural".

Yanina Fantasía en Cuando el aula tradicional se diluye... la educación a distancia en la Licenciatura en Enfermería, relata la experiencia de una carrera de grado donde una plataforma virtual da lugar a un entramado de educación e inclusión social.

En el quinto capítulo, Susana Marchisio, en Una historia de compromisos, legados y utopías en la búsqueda de caminos para la democratización del saber, describe los inicios y la evolución de la modalidad en la Facultad de Ciencias Exactas, Ingeniería y Agrimensura, así como la significativa integración de la Red Universitaria de Educación a Distancia, RUEDA. Esta emerge como un ámbito institucional cuyo rol trasciende el análisis sobre la modalidad como alternativa pedagógica, para constituirse en un espacio de debate y discusión que asume a la educación a distancia como canal insoslayable de articulación entre la producción de conocimiento y la democratización de la educación.

Marcela Rosales en el sexto capítulo, hace referencia a la incidencia de los cambios tecnológicos, que junto a lo metodológico y conceptual confluyen en una cátedra de la carrera de Comunicación Social. La incidencia de las transformaciones acaecidas a fines de los 90 , tanto en el entorno sociotecnológico como en las prácticas comunicacionales, permite visualizar un escenario que una década después, irrumpiría definitivamente en los modos de relación entre alumnos, docentes y entorno.

En Un encuentro necesario. Experiencias compartidas en la Educación a Distancia en la Universidad Nacional de Rosario, Gaspar Aita, relata una experiencia de relacionamiento interinstitucional, donde las capacidades desarrolladas por el equipo de Educación a Distancia de la Facultad de Ciencias Exactas, Ingeniería y Agrimensura se sinergizan con los saberes de Psicología Educativa I de la carrera de Sicología favoreciendo la articulación entre los contenidos de la presencialidad y la modalidad virtual. 
Finalmente, en el último capítulo, Susana Copertari, Natalia Sgreccia y María Laura Segura, a través de un recorrido por los casos de educación a distancia en las carreras de posgrado de la UNR, logran realizar una síntesis acabada de la situación de esta universidad donde, desde modalidades mixtas o semipresenciales, las aulas virtuales o campus ofrecen alternativas de interacción a estudiantes y docentes.

Desde un relato, que detalla diversas experiencias en torno a la construcción de una pedagogía desde la virtualidad en la educación superior y en el marco de una fundamentación teórica profunda, los autores logran adentrarnos a través de esta obra en la complejidad de la modalidad y su implementación.

La Educación a Distancia se nos presenta como una estrategia educativa que se sustenta en el uso racional y adecuado de las nuevas tecnologías, estructuras operativas flexibles y métodos pedagógicos altamente eficientes en la enseñanza-aprendizaje. Se materializa en el análisis de experiencias que, en una relación dialógica, logran conjugar planes de estudio, redacción de contenidos, diseño de actividades y espacios virtuales atravesados por las diversas tecnologías, con un nuevo modo de comunicar e interactuar. Desde conceptualizaciones, reflexiones y relatos, los autores contribuyen a "diseñar situaciones de enseñanza donde la distancia se convierte en una categoría que condiciona favorablemente las decisiones pedagógicas" tal como lo proponen Copertari y Morelli.

El recorrido histórico realizado, nos lleva a reflexionar sobre una dimensión tecnológica que trasvasa las distintas disciplinas y que se presenta como alternativa al permitir que los factores de tiempo, espacio, ocupación o edad de los estudiantes no se conviertan en elementos limitantes o condicionantes de la introducción, retención y utilización de la información.

Finalmente, es una obra que transciende la Educación a Distancia en la Universidad Nacional de Rosario como modelo pedagógico. Logra conducirnos a repensar los modos de organización y gestión del conocimiento como respuesta a los problemas sociales contemporáneos desde una lógica donde las dimensiones académicas, institucionales, culturales, administrativas y políticas, no están ausentes. 